\title{
Aplicación de la técnica LSPIV en el diseño hidrológico de infraestructura hídrica
}

\section{LSPIV application in the hydrological design of hydraulic infrastructure \\ Myriam Cecilia Botelli', Nicolás Federico Guillén ${ }^{2}$, Carlos Marcelo García ${ }^{2}$, Antoine Patalano ${ }^{2}$ y Marcelo Chalabe ${ }^{1}$}

Ingeniería Civil / artículo científico

Citar: Botelli, M. C., Guillén, N. F., García, C. M., Patalano, A. y Chalabe, M. Aplicación de la técnica LSPIV en el diseño hidrológico de infraestructura hídrica. Cuadernos de Ingeniería, 12. Recuperado de http://revistas.ucasal.edu.ar

Recibido: marzo/2020

Aceptado: julio/2020

\section{Resumen}

En este trabajo se implementa una nueva técnica de medición de caudales en la provincia de Salta. Los flujos que ocurren durante crecidas repentinas en ríos de montaña, (como lo son la mayoría de los casos en Salta), presentan algunas características particulares que hacen que tecnologías intrusivas de velocimetría (tales como molinetes convencionales o correntímetros hidroacústicos Doppler ADCP) habituales para medir caudales en cursos fluviales no puedan ser implementadas. En el presente estudio se evalúa la aplicación de la técnica experimental remota LSPIV (Large Scale Particle Image Velocimetry) para medir velocidades de flujo en ríos de la provincia de Salta, Argentina. Para ello, se instalaron estaciones meteorológicas en zonas de interés y se estimaron recurrencias de los eventos de crecidas caracterizadas.

Palabras clave: LSPIV, crecidas, hidrología de diseño, hidráulica

\section{Abstract}

In this work, a new flow measurement technique is implemented in the province of Salta. The flows that occur during flash floods in mountain rivers, (as most of the cases in Salta), have some particular characteristics that make intrusive technologies of velocimetry (such as conventional hydraulic reels or Doppler hydroacoustic flowmeters

\footnotetext{
1 Facultad de Ingeniería, Universidad Católica de Salta (UCASAL), Argentina.

2 Facultad de Ciencias Exactas, Físicas y Naturales, Universidad Nacional de Córdoba (UNC), Argentina.
} 
- ADCP) usual to measure flows in river courses cannot be implemented. In the present study, the application of the remote experimental technique LSPIV (Large Scale Particle Image Velocimetry) to measure flow velocities in rivers of Salta province, Argentina, is evaluated. For this purpose, meteorological stations were installed in areas of interest and recurrences of characterized flood events were estimated.

Keywords: LSPIV, flash floods, design hydrology, hydraulics

\section{Introducción}

El manejo sustentable del recurso hídrico requiere tener una cuantificación precisa de la disponibilidad de ese recurso. Sin esta información, los planes de gestión y manejo no serán eficientes. En la actualidad, la disponibilidad espacial y temporal de cuantificaciones del recurso hídrico superficial no es habitual en la República Argentina; en especial en áreas áridas y semiáridas del país, donde el recurso hídrico es escaso, se presentan falencias importantes en monitoreos realizados durante la ocurrencia de crecidas.

Los ríos de la provincia de Salta, Argentina, se caracterizan por la ocurrencia de inundaciones repentinas con gran volumen de escurrimiento durante la época de lluvias (octubre a abril). Debido a la naturaleza repentina de estas inundaciones, es muy poco probable que los técnicos e investigadores sean capaces de inspeccionar varios tramos fluviales de interés utilizando los instrumentos y las técnicas usuales (molinetes convencionales o correntímetros hidroacústicos Doppler) para el registro del caudal máximo y su evolución temporal durante la inundación. En caso de que sea posible llegar a tiempo a los sitios de estudio, las velocidades de flujo y los objetos flotantes en el río (por ejemplo, troncos) ponen en peligro tanto a los operadores como a los instrumentos.

En la actualidad, se encuentran en continuo desarrollo las técnicas experimentales denominadas técnicas de velocimetría no intrusivas por imágenes digitales, Particle Image Velocimetry (PIV por sus siglas en inglés). PIV ha sido ampliamente utilizada en experimentos de laboratorio en la hidráulica por más de 30 años (Adrian, 2005, 1991, 1984), y desde la década del 80, muchos investigadores intentaron adaptar la técnica para caracterizar flujos de mayores escalas a los presentes en laboratorio, incluyendo mediciones de campo, lo que generó la técnica Velocimetría por Imágenes de Partículas a Gran Escala (LSPIV por sus siglas en inglés Large Scale Particle Image Velocimetry) (Creutin et al., 2003, Fujita et al., 1998, Gunawan et al., 2012, Le Coz et al., 2014, Muste et al., 2008).

LSPIV es una moderna técnica experimental con gran potencial para el monitoreo de diversos flujos superficiales en cauces naturales de manera remota (no intrusiva). Con esta técnica se puede analizar el desplazamiento de las partículas que flotan sembradas en el flujo, o determinados patrones que existen sobre la superficie del agua, generados por la turbulencia. El desarrollo de esta técnica en los últimos años ha permitido su aplicación en la caracterización de flujos complejos con avances notables en el campo de la mecánica de los fluidos e hidráulica. En este sentido, investigadores de la Universidad Nacional de Córdoba se encuentran trabajando perma- 
nentemente en la mejora de los programas computacionales que permiten la aplicación de LSPIV y posibilitan cuantificar el caudal que escurre por un curso fluvial a partir del registro y análisis de un simple video de la superficie del agua y de algunos relevamientos topográficos que se realizan posteriormente al evento. Los nuevos medios de comunicación y los avances tecnológicos permiten que los ciudadanos generen una gran cantidad de material digital sobre las crecidas repentinas y las compartan a través de las redes sociales. Este tipo de información se presenta a menudo con autoría, fecha y, en unos casos, georreferenciada, antes de ser compartida.

El objetivo de este trabajo es aplicar la técnica LSPIV para cuantificar caudales en ríos de la provincia de Salta durante eventos extremos y estimar su recurrencia asociada.

\section{Metodología}

Las técnicas de velocimetría por imágenes digitales más conocidas son las técnicas de velocimetría por imágenes clásicas: la Velocimetría por Seguimiento de Partículas (PTV, por sus siglas en inglés), la Velocimetría por Imágenes de Partículas (PIV, por sus siglas en inglés) y su implementación a gran escala (LSPTV y LSPIV, respectivamente, por sus siglas en inglés). Estas técnicas podrían ser utilizadas para generar información que se requiere para el diseño hidrológico e hidráulico de medidas estructurales y no estructurales y así mitigar el riesgo hídrico.

Las técnicas de medición por imágenes permiten caracterizar el campo de velocidades de flujos con alta resolución temporal y espacial mediante un análisis de imágenes que ilustran los desplazamientos de trazadores (partículas sembradas o patrones superficiales) que caracterizan el flujo en el sector analizado. Una de esas técnicas denominada Velocimetría por Imágenes de

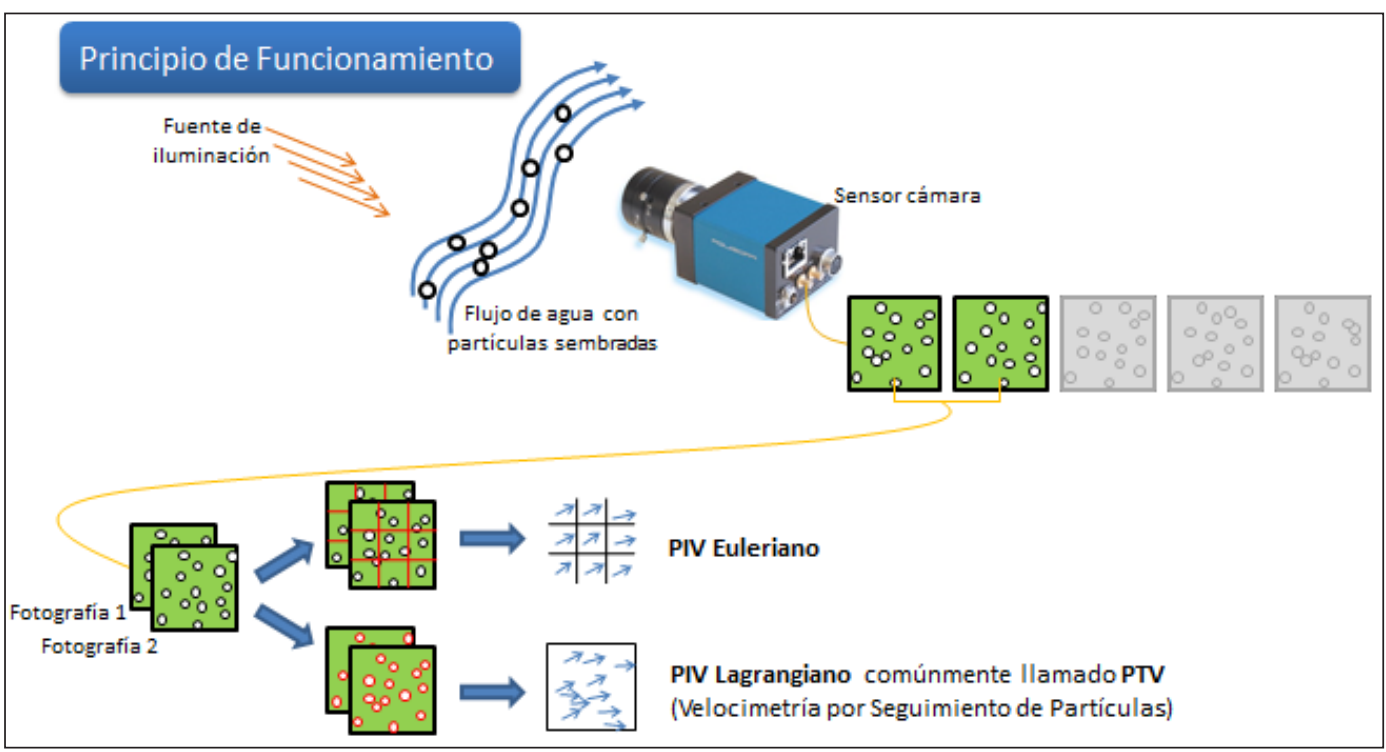

Figura 1: Esquema del conjunto de elementos para la aplicación de PIV/PTV clásico 


\section{C. Botelli et al.}

Partículas PIV (Particle Image Velocimetry) prevé el análisis euleriano del desplazamiento de los trazadores, debido a que obtiene los campos de velocidades del flujo comparando el desplazamiento de patrones o grupos de partículas en un sector definido de una imagen; en cambio, la Velocimetría de Seguimiento de Partículas PTV (Particle Tracking Velocimetry) prevé el análisis lagrangiano, ya que analiza cada partícula en forma individual y determina su desplazamiento al comparar su posición en distintos momentos del tiempo. En la Figura 1 se muestra un esquema del funcionamiento de las técnicas PIV/PTV. La metodología experimental general consiste en obtener un video (con una longitud de registro suficiente para obtener parámetros estadísticos representativos) que permita evaluar el desplazamiento de trazadores dentro del flujo con una adecuada iluminación.

Para la estimación de las velocidades del flujo en gabinete se necesita una computadora con los siguientes programas instalados:

- PIVlab (se descarga de http://pivlab.blogspot.com.ar/, Thielicke y Stamhuis, 2014). Este software de código abierto permite la aplicación de PIV clásico.

- RIVeR (se descarga de http://riverdischarge.blogspot.com.ar/, Patalano et al., 2017). Es una herramienta que ha sido desarrollada desde el 2013 por el Centro de Estudios y Tecnología del Agua (CETA) de la Universidad Nacional de Córdoba (UNC) para la aplicación de LSPIV.

\section{Resultados de la aplicación de la técnica LSPIV en la Provincia de Salta}

A continuación, se presentan diferentes aplicaciones y resultados alcanzados, producto de la implementación de la técnica experimental remota LSPIV en la provincia de Salta.

\subsection{Uso de LSPIV para cuantificar un evento hidrológico extremo con videos genera- dos por colaboración abierta distribuida}

A continuación, se detalla la cuantificación del escurrimiento superficial extremo generado en el río Colorado, cercano a la localidad de Orán, durante el evento hidrometeorológico extraordinario del 28 de marzo de 2017. Esta cuantificación se hizo a partir del análisis de un video enviado por un ciudadano.

Entre la última semana de marzo y la primera de abril de 2017, según diferentes datos recopilados, cayeron en la zona del río Colorado unos 350 milímetros. En el proyecto de investigación anterior elaborado por el grupo de investigación autor de este trabajo, se estimaron las láminas de lluvia precipitadas en 15 días consecutivos en la estación Bermejo Pozo Sarmiento, muy cercana a la zona de estudio. Al analizar los resultados presentados en la Figura 2, se observa que el evento hidrometeorológico que se busca aquí caracterizar tiene una recurrencia mayor a los 10 años. 
Aplicación de la técnica LSPIV en el diseño hidrológico de infraestructura hídrica

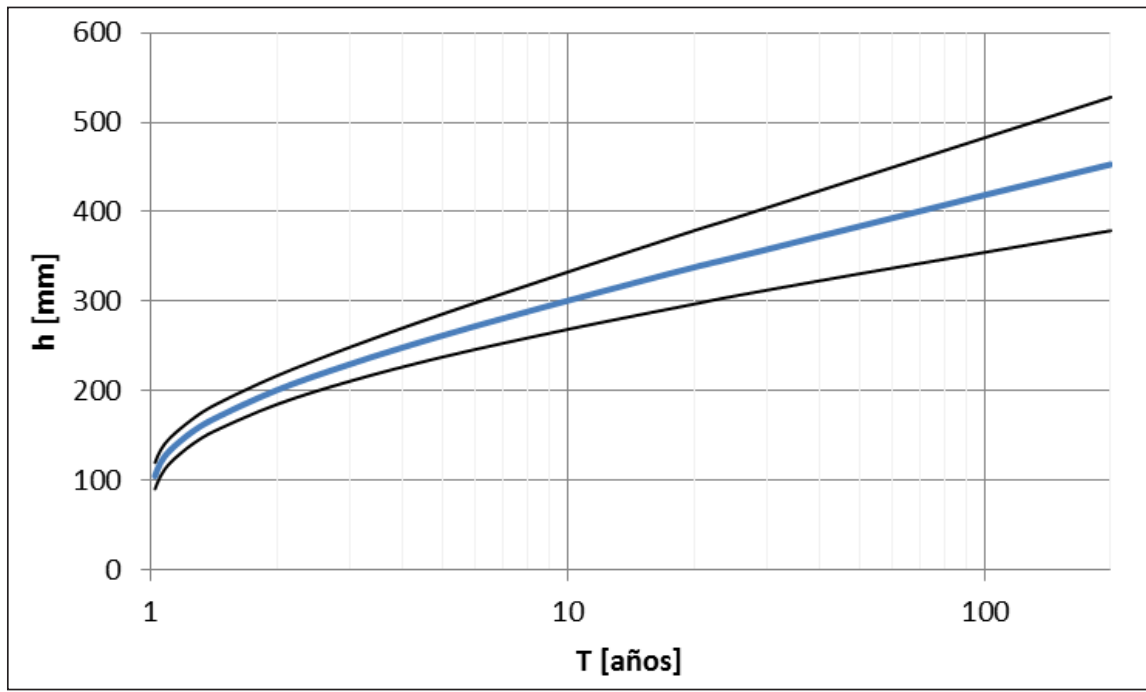

Figura 2: Láminas de lluvia precipitadas en 15 días consecutivos, asociadas a diferentes recurrencias en estación Bermejo - Pozo Sarmiento

Para caracterizar la crecida extraordinaria, se detallan las estimaciones realizadas a partir de la modelación numérica de la hidrodinámica del flujo del río cuando cruza el puente de la Ruta Nacional N. ${ }^{\circ}$ 0, aplicando el programa HEC RAS. En las etapas de calibración e implementación del modelo numérico, se utilizaron datos relevados en la zona del estudio (velocidades superficiales del flujo, alturas de la superficie libre, etc.). Esta información fue utilizada para la calibración de modelos hidrológicos e hidráulicos; los resultados de este análisis son la única información hidrológica disponible para ese evento.

A partir de datos de relevamientos topográficos, se elaboró un modelo digital de elevación del terreno a partir del cual se determinó la geometría del dominio donde se implementó el modelo hidráulico HEC RAS. Se contaba con una serie de 5 perfiles transversales aguas arriba de la sección del puente y otros 5 perfiles aguas abajo. Las condiciones de borde empleadas en el modelo fueron las de tirante normal tanto aguas arriba como aguas abajo. Para la implementación del modelo fue necesario definir parámetros o coeficientes de rugosidad que se requieren para simular la resistencia al flujo del fondo y las márgenes. Los coeficientes de rugosidad adoptados en este estudio (coeficientes de rugosidad «n» definidos por Manning) se detallan a continuación:

- Se adoptó un valor de «n» de Manning de 0,035 para el cauce (valor extraído de la bibliografía para ríos similares al analizado).

- Para determinar el coeficiente de las planicies de inundación se implementó una metodología novedosa. En primer lugar, se analizó un video digital de la crecida, registrado por un vecino, en una sección conocida del tramo de estudio con el fin de estimar la velocidad superficial del flujo mediante la aplicación de LSPIV. Además, a partir de este video se determinaron las alturas de la superficie libre de flujo alcanzadas. A partir de la velocidad y la altura 
del flujo estimadas del análisis del video digital se calibró el valor del «n» de Manning de la planicie (igual a 0,05). En las Figuras 3 y 4 se observan los principales resultados alcanzados con LSPIV y se presenta el campo de velocidad de flujo en la sección de control obtenido con los valores de los «n» de Manning estimados como se explicó. Se observa una velocidad en la margen derecha de $2,75 \mathrm{~m} / \mathrm{s}$ y una altura de la superficie libre unos centímetros por debajo de la cota inferior de la viga del puente. La resolución de las imágenes digitales registradas no era óptima para aplicar la técnica de velocimetría LSPIV en la región central y la margen izquierda del cauce (alejada de la cámara).
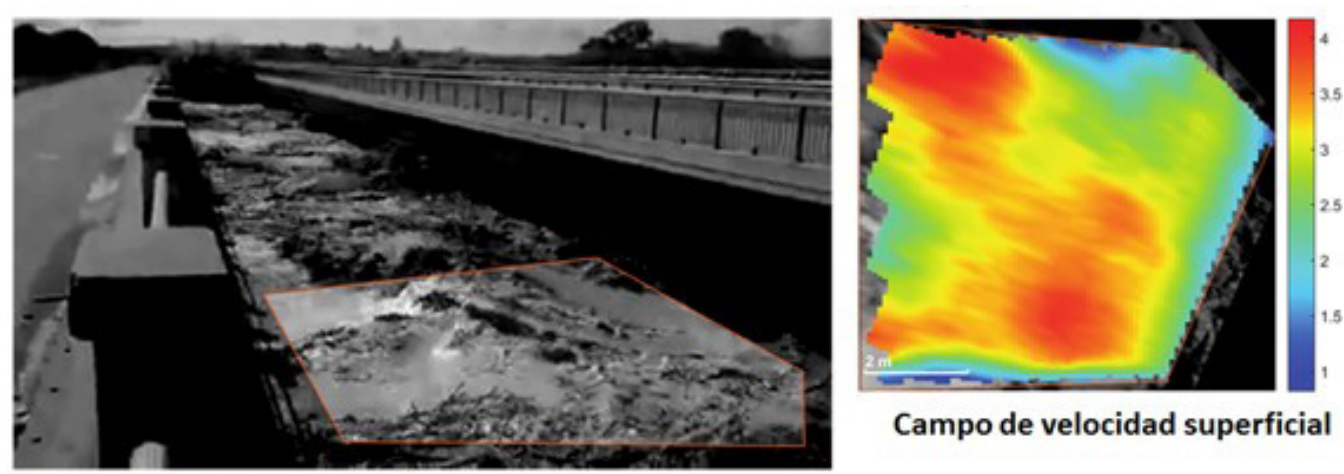

Campo de velocidad superficial

\section{Región analizada con LSPIV}

Figura 3: Región donde se estimaron velocidades y campo de velocidad media superficial estimada con LSPIV, en el video captado en el puente que cruza el río Colorado en la Ruta Nacional N. ${ }^{\circ}$ 50, Orán, Salta

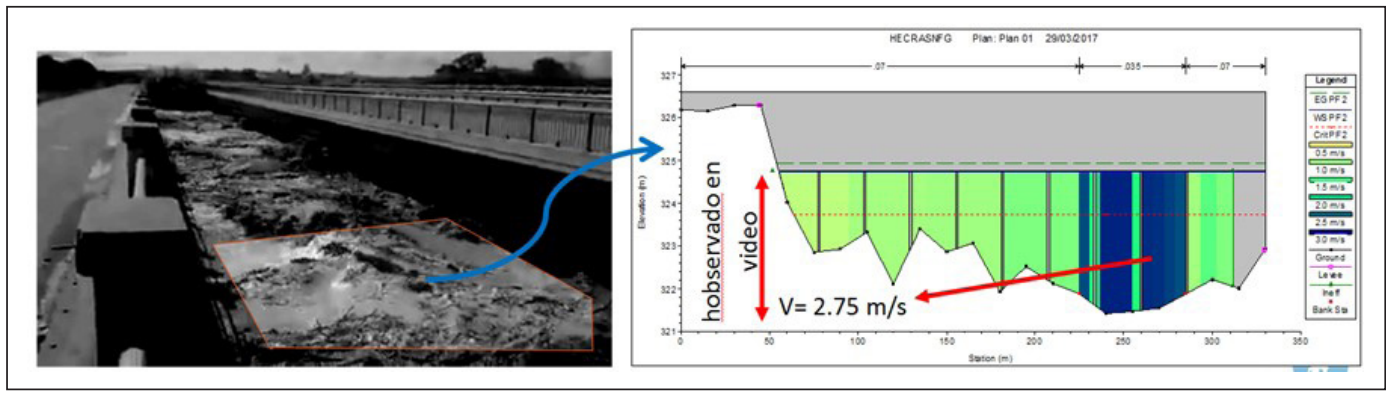

Figura 4: Sección de control para la calibración del «n» de Manning

Se procedió a la modelación hidrodinámica del tramo del cauce y se estimó un caudal escurrido durante el registro del video de $850 \mathrm{~m}^{3} / \mathrm{s}$. En la Figura 5 se muestran las áreas inundadas obtenidas a partir de la simulación numérica realizada, y se observa claramente que el flujo escurre en todo el tramo simulado con régimen subcrítico (ya que el tirante alcanzado es superior al crítico — que se marca con línea punteada roja— en todo el tramo). 


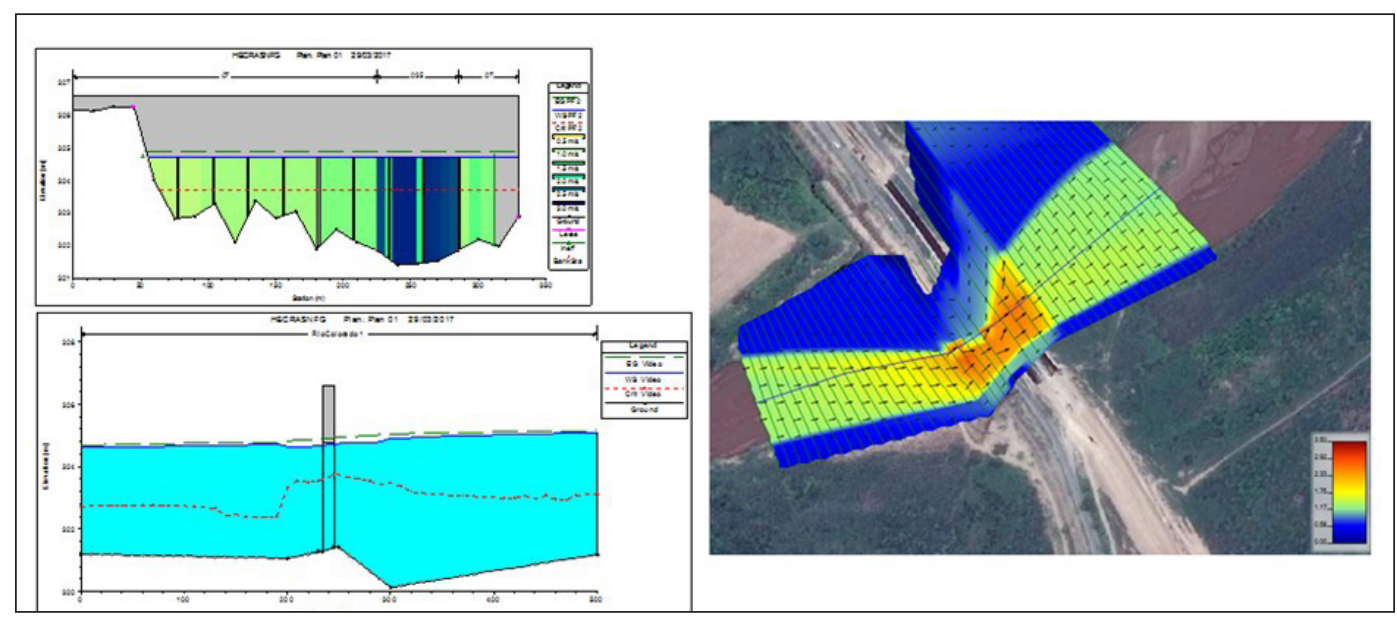

Figura 5: Áreas inundadas obtenidas a partir de la simulación numérica realizada para un caudal de $850 \mathrm{~m}^{3} / \mathrm{s}$

Para contrastar los resultados alcanzados, posteriormente se ingresaron al modelo los caudales de diseño previamente calculados por el Ing. Néstor Ilvento, un profesional especialista en la temática y referente de la provincia de Salta. Los valores ingresados para evaluar el funcionamiento del puente corresponden a tiempos de retorno de 50 y 100 años, y son iguales a 1690 y $1940 \mathrm{~m}$ 3/s, respectivamente. A continuación, se presentan los principales resultados:

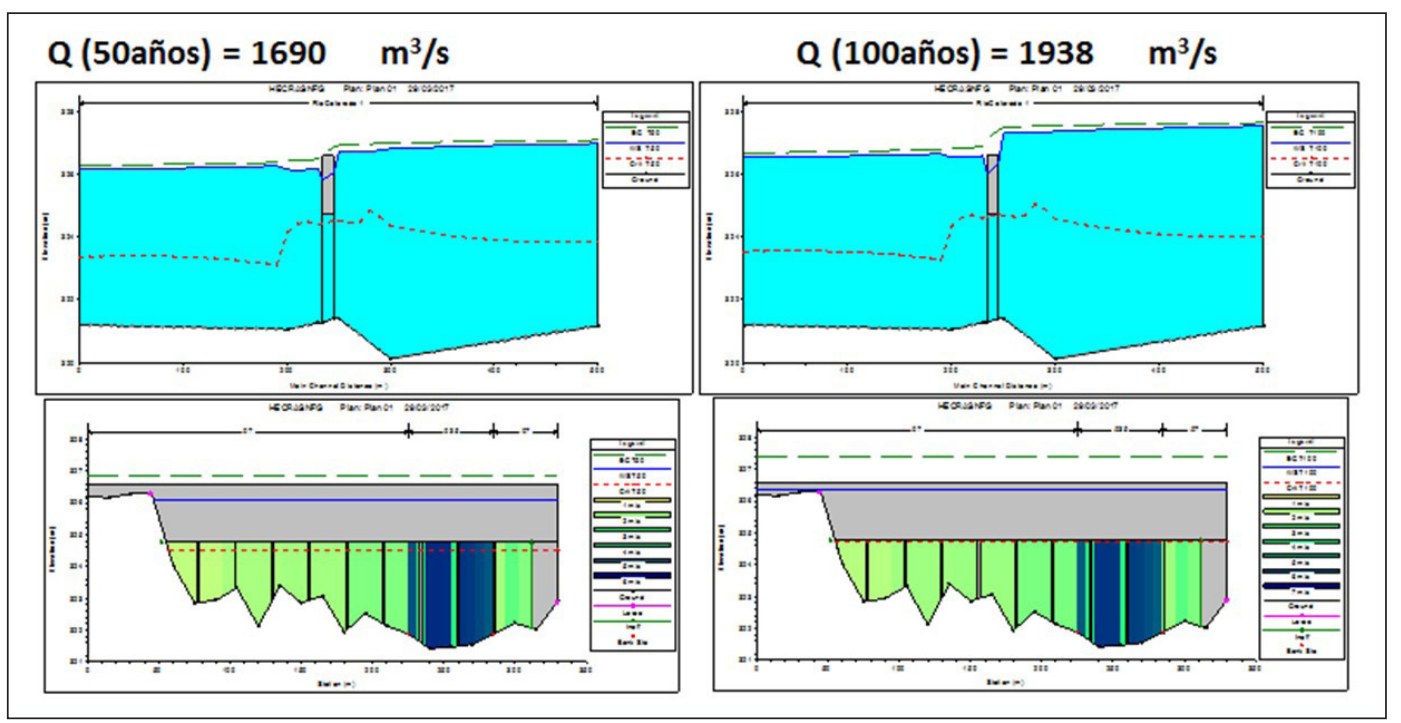

Figura 6: Modelación hidráulica de los caudales de diseño asociados a 50 y 100 años en el tramo del estudio del río Colorado 
$\mathrm{Al}$ analizar los resultados alcanzados, se observa que la cota inferior de la viga del puente se ve ampliamente superada para los caudales de diseño.

Con la implementación de LSPIV se obtuvo información fundamental para la calibración del modelo hidrodinámico que simula el evento ocurrido, lo que se logró con la determinación del coeficiente de rugosidad, antes mencionado, a partir de la velocidad superficial del flujo obtenida por LSPIV.

Por otra parte, con este estudio se determinaron las alturas de la superficie libre de flujo alcanzadas y se pudo demostrar que el nivel de agua supera la cota de viga del puente para las crecidas de diseño, por lo que no fue debidamente diseñado.

A futuro, antes del diseño de una obra hídrica, sería fundamental contar con aforos de las crecidas extraordinarias de esa localización, y LSPIV es una técnica adecuada para casos como el aquí presentado.

\subsection{Ajuste de curvas altura del nivel de agua - caudal (H-Q) en diferentes cauces flu- viales de la provincia de Salta}

Se realizaron múltiples campañas en diferentes cauces de la provincia de Salta, en el periodo 2019/2020, para la estimación de alturas H-Q. A continuación, se presentan a modo de ejemplo los resultados de una de las campañas de medición y las H-Q ajustadas.

El miércoles 29 de enero de 2020, se realizaron mediciones en los afluentes del dique Cabra Corral. Además de captar videos de alta resolución de cada uno de los cauces, se relevaron distancias entre puntos fijos que se observan en el video (para poder rectificar posteriormente los resultados) y también se relevó la cota del pelo de agua al momento del aforo. Seguidamente, se presentan los principales resultados alcanzados.

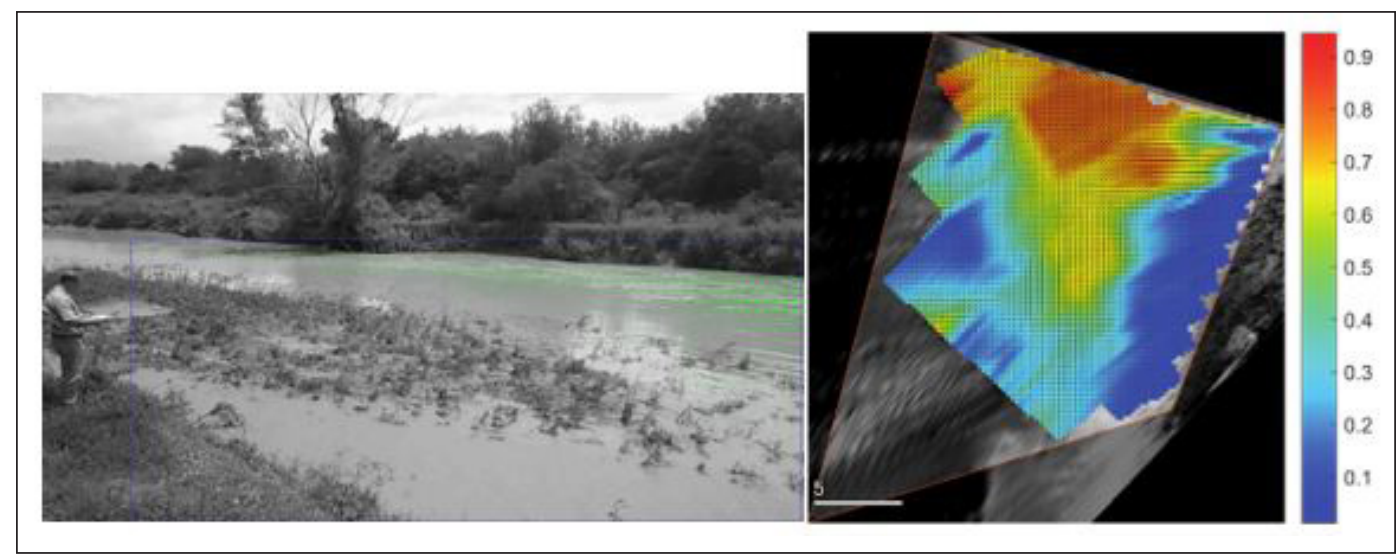

Figura 7: Vectores velocidad (a la izquierda) y campo de velocidades superficiales con su respectiva zona de medición (a la derecha) en la sección de control del río Arenales 
Aplicación de la técnica LSPIV en el diseño hidrológico de infraestructura hídrica

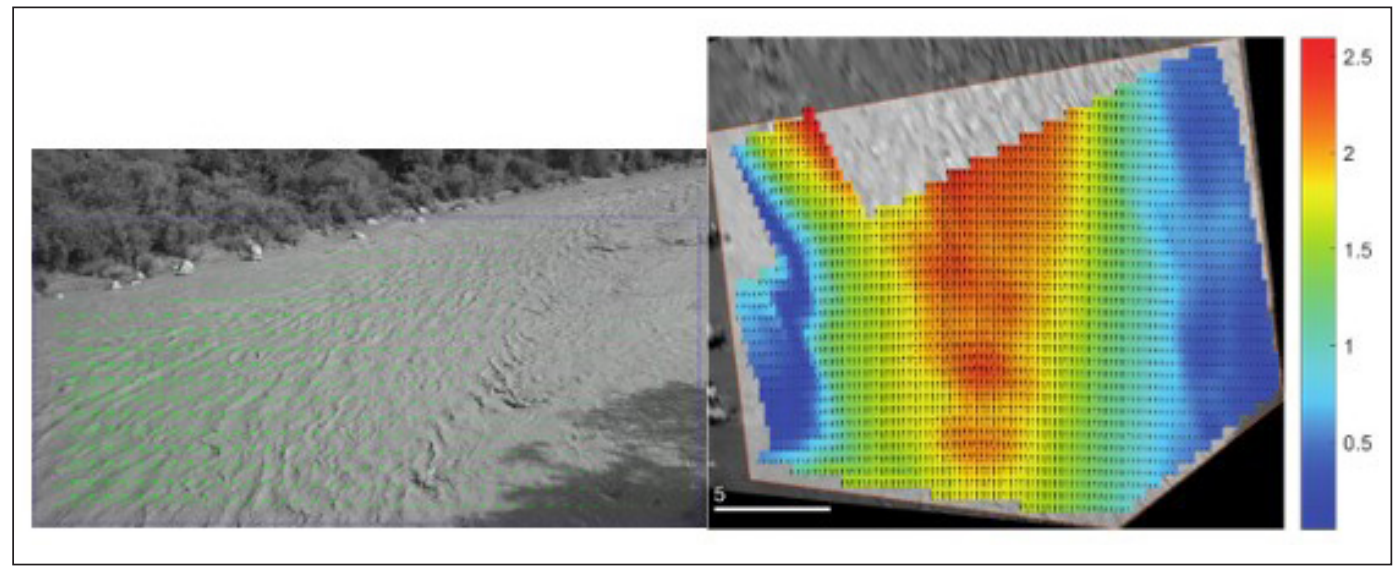

Figura 8: Vectores velocidad (izquierda) y campo de velocidades superficiales (derecha) en la sección de control del río Guachipas

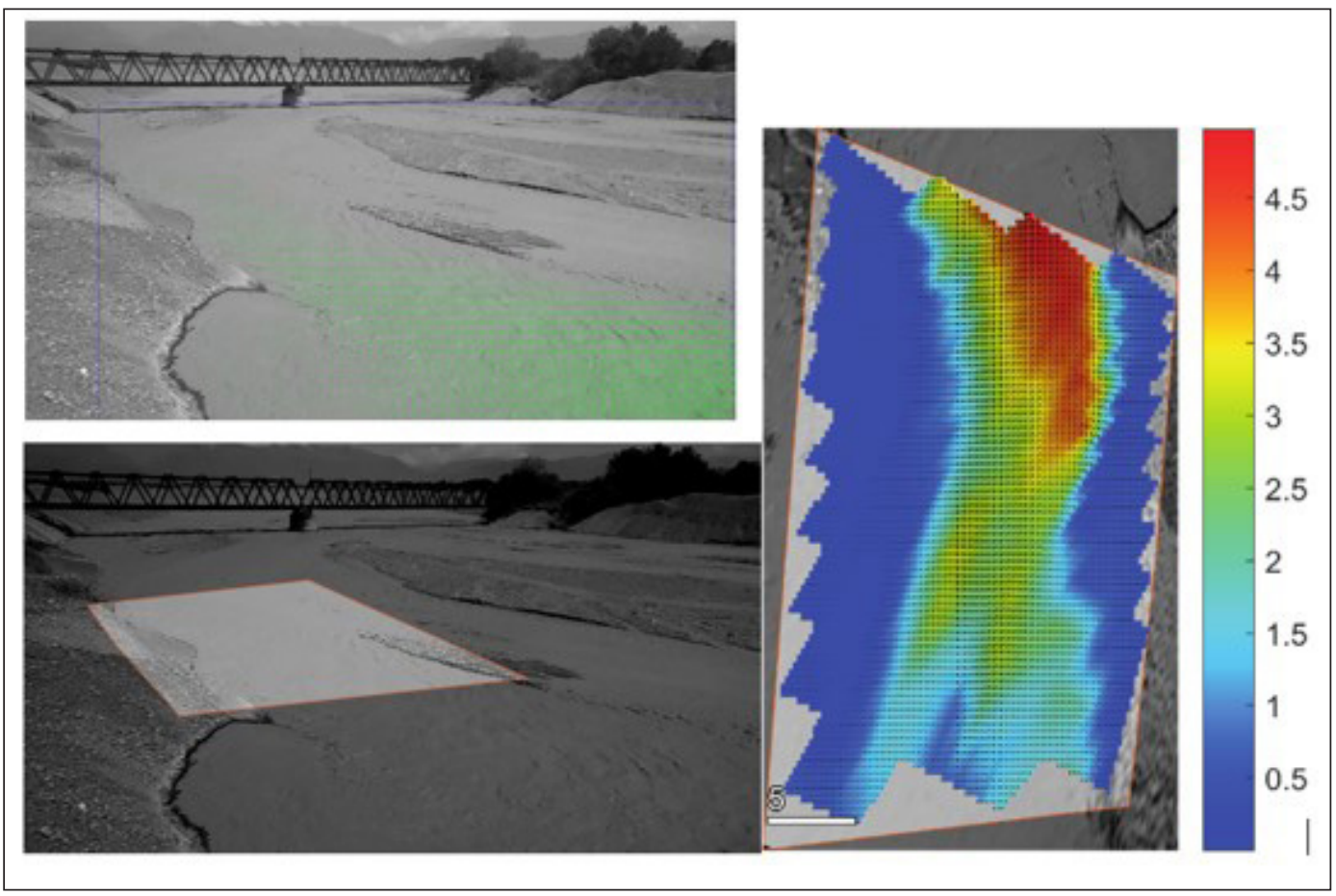

Figura 9: Vectores velocidad (arriba) y campo de velocidades superficiales con la zona rectificada (abajo) en la sección de control del río Rosario, filmado desde la margen derecha 
Durante la campaña de medición, la técnica experimental de aforo funcionó muy adecuadamente en la detección de las velocidades superficiales del flujo.

Para la construcción de las curvas H-Q, posteriormente al aforo de algunos eventos en los cauces de estudio, se procedió al desarrollo de un modelo hidráulico calibrado en cada uno de los ríos de interés. Para ello se realizaron relevamientos topográficos de detalle y una serie de 10 perfiles transversales en cada uno de los sitios de estudio. Las condiciones de borde empleadas en el modelo fueron las de tirante normal tanto aguas arriba como aguas abajo.

Seguidamente, se presentan los modelos hidráulicos con los coeficientes de rugosidad calibrados, de tal manera que para las cotas de pelo de agua medidas en campo coincide la velocidad superficial medida in situ con la estimada por el modelo hidráulico HEC RAS:

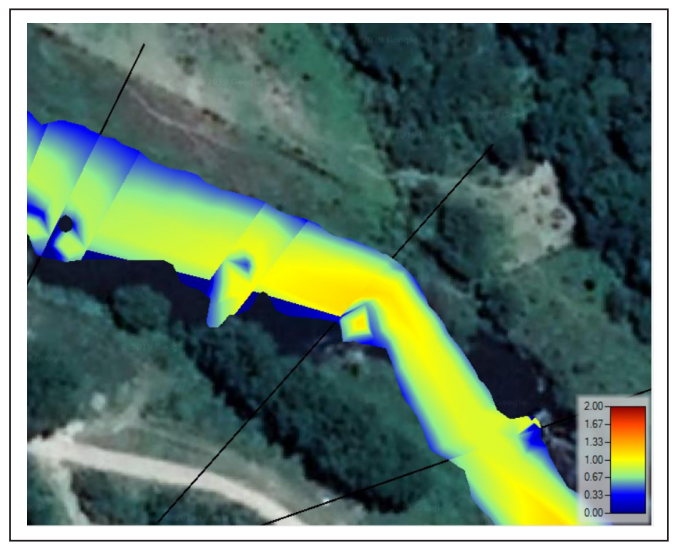

Figura 10: Campo de velocidades estimado con HEC RAS en el río Arenales

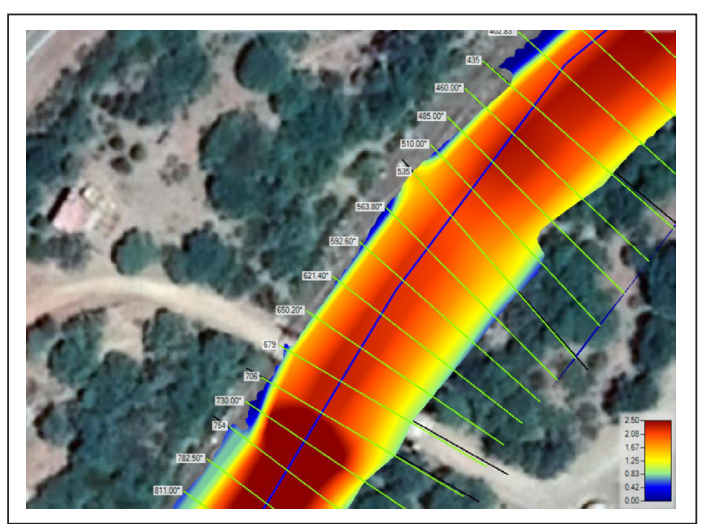

Figura 11: Campo de velocidades estimado con HEC RAS en el río Guachipas

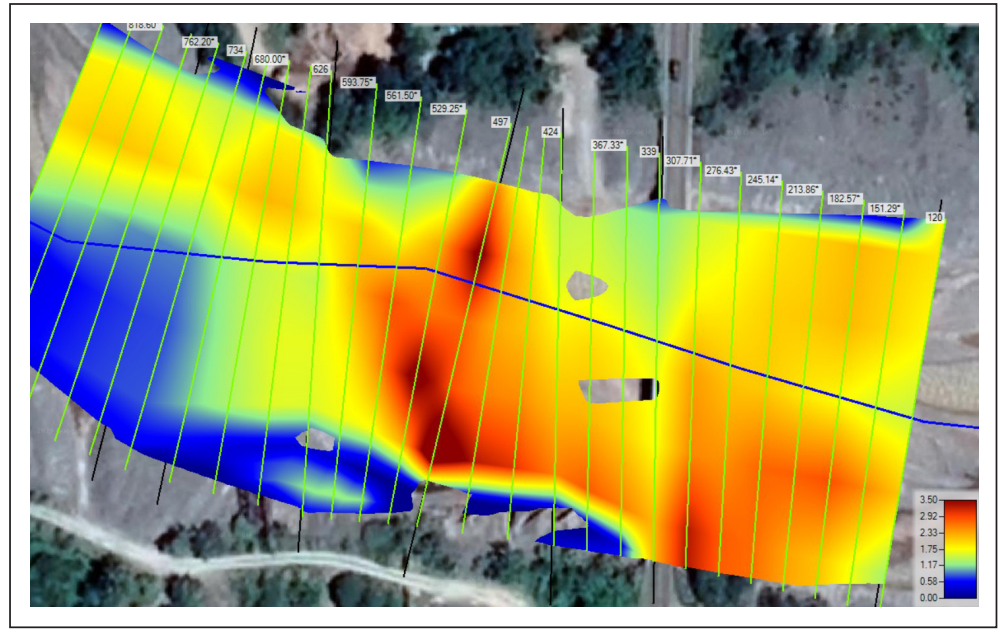

Figura 12: Campo de velocidades estimado con HEC RAS en el río Rosario 
Los caudales escurridos estimados durante la campaña de medición en los diferentes cauces aforados son los siguientes:

- Río Arenales: $10 \mathrm{~m}^{3} / \mathrm{s}$

- Río Rosario: $100 \mathrm{~m}^{3} / \mathrm{s}$

- Río Guachipas: $50 \mathrm{~m}^{3} / \mathrm{s}$

- Río Chuñapampa: $5 \mathrm{~m}^{3} / \mathrm{s}$

Finalmente, luego de realizar múltiples campañas de aforo se ajustaron las siguientes relaciones H-Q en los cauces presentados:

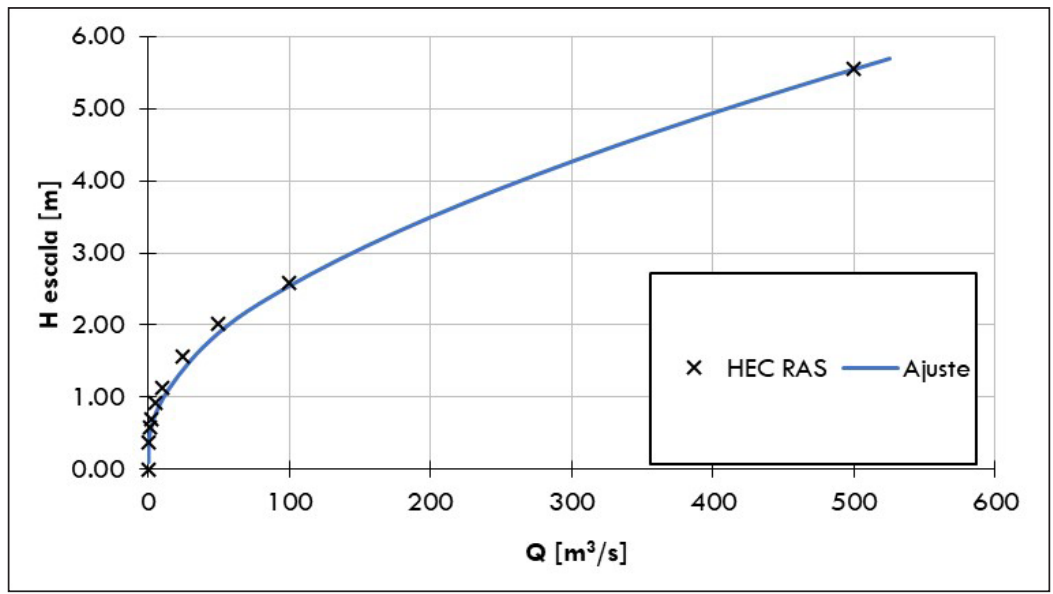

Figura 13: Curva H-Q en el río Rosario en el cruce con la Ruta Nacional N. ${ }^{\circ}$ 68, provincia de Salta

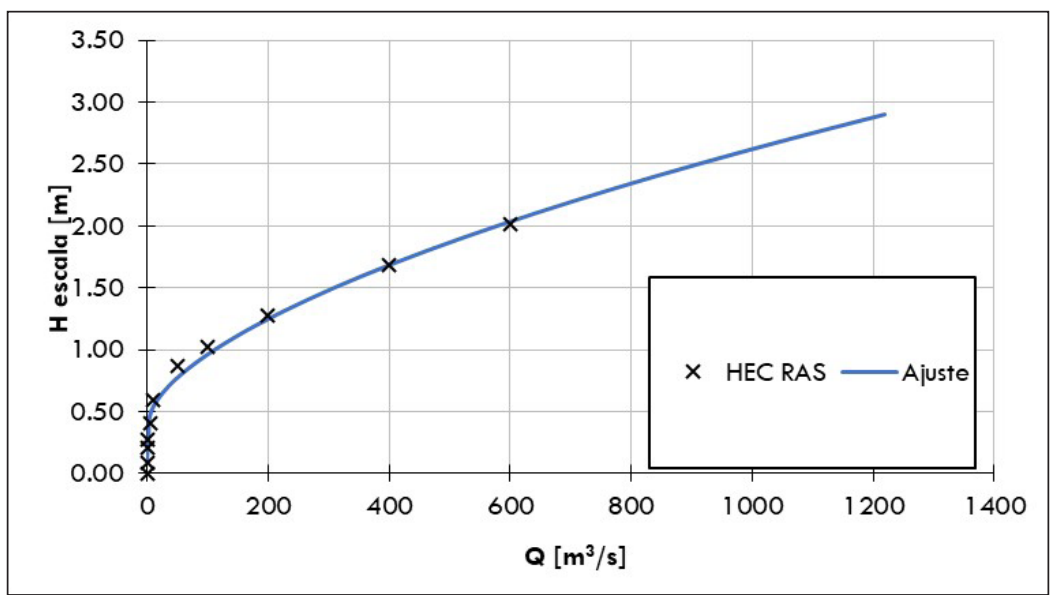

Figura 14: Curva H-Q en el río Arenales cercano a la localidad de San Agustín, provincia de Salta 


\section{C. Botelli et al.}

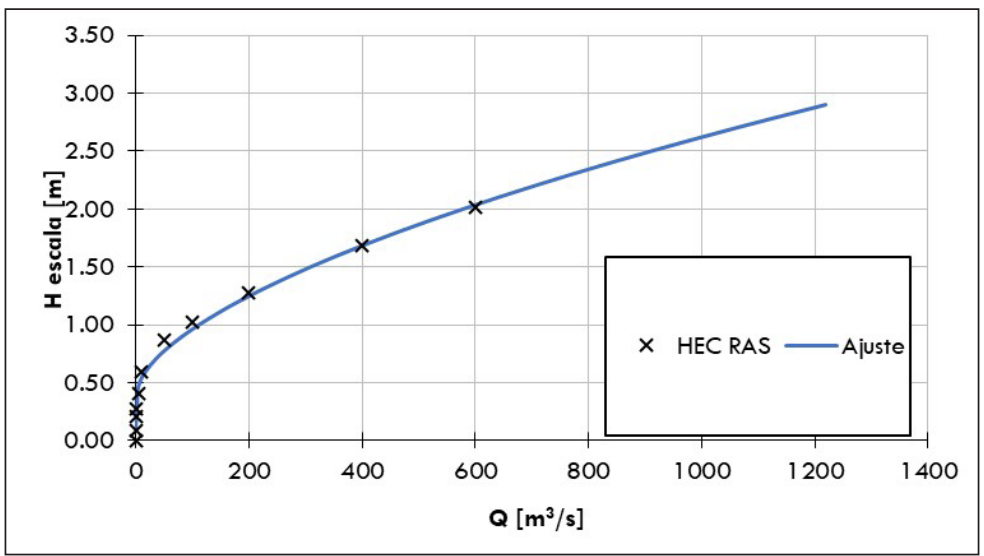

Figura 15: Curva H-Q en el río Guachipas en el ingreso a la localidad de Alemania, provincia de Salta

\subsection{Evaluación de la velocidad y la concentración del flujo denso en el Río Chuscha, Provincia de Salta}

En este punto se presenta el análisis realizado para caracterizar las condiciones de flujo denso presentes en la sección definida en el río Chuscha, en su intersección con la ruta nacional $\mathrm{N}{ }^{\circ}$ 40, en la zona norte de la ciudad de Cafayate, provincia de Salta. El evento analizado corresponde a un evento de inundación repentina, ocurrido el 6 de enero de 2015. Durante el evento un vecino registró un video (una imagen se observa en la Figura 16) que se analiza en este apartado.

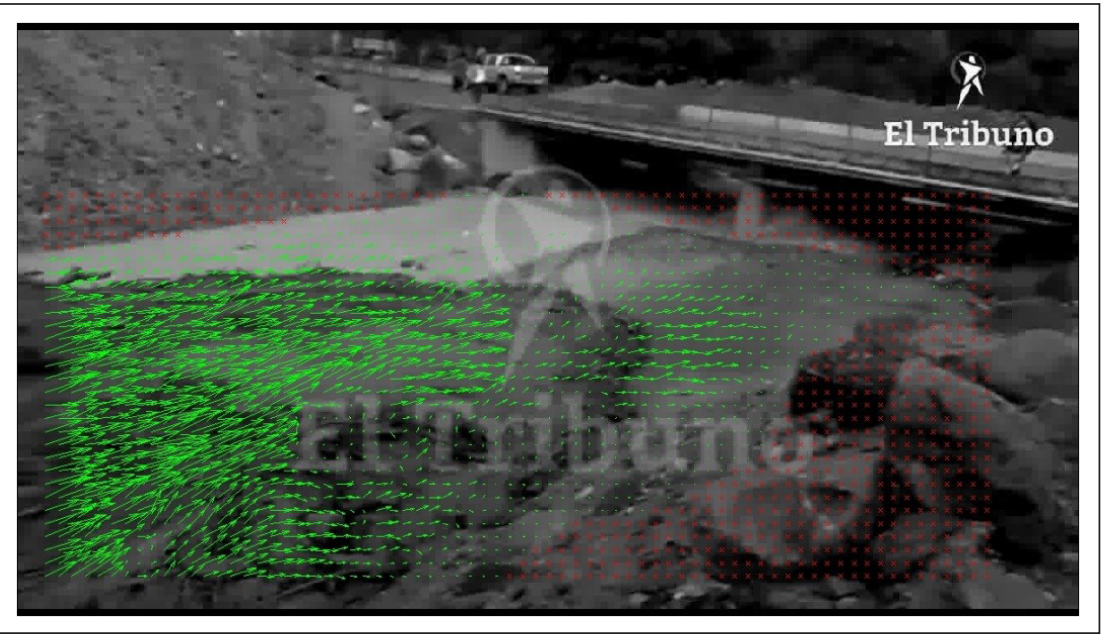

Figura 16: Campo de vectores desplazamiento medio al aplicar LSPIV 
Utilizando las distancias relevadas in situ y el campo de desplazamiento medio, se estimó que la velocidad del frente media de la sección es de $3 \mathrm{~m} / \mathrm{s}$. Luego de la implementación de LSPIV para la estimación de las velocidades del frente del flujo denso, se busca estimar la concentración del sedimento del flujo mediante modelos existentes en la bibliografía.

Lo que estos modelos calculan es la velocidad del frente del flujo denso, por lo que se realizó el camino inverso: es decir, se buscó cuál era la concentración volumétrica del sedimento que hacía que la velocidad del frente fuera del mismo orden que la estimada con LSPIV previamente.

$\mathrm{Al}$ asumir una concentración volumétrica del sedimento del $10 \%$, se obtuvieron las siguientes velocidades de frente del flujo denso al aplicar los modelos descriptos en la Tabla 1.

Tabla 1: Velocidades de frente del flujo denso al aplicar los modelos propuestos por Takahashi (1981) al utilizar una concentración volumétrica del sedimento de $10 \%$.

\begin{tabular}{|l|c|}
\hline Modelo & {$[\mathrm{m} / \mathrm{s}]$} \\
\hline Bagnold (1954) & 3,00 \\
\hline Yano y Daido (1965); Johnson (1970) & 2,86 \\
\hline
\end{tabular}

Al comparar la velocidad experimental obtenida en este trabajo a partir de la implementación de LSPIV con los valores calculados por las fórmulas propuestas por los diferentes modelos, se observa que los modelos aplicados estiman velocidades de frente de flujo denso muy cercanas a la medida experimentalmente con LSPIV al asumir una concentración volumétrica del sedimento del $10 \%$.

Lo que aquí se presenta cubre un área vacante, ya que en el pasado no ha sido posible medir in situ velocidades de frentes de flujos densos; de aquí en adelante mediante la aplicación de LSPIV podrá ser posible no solamente la medición de las velocidades de estos flujos, sino también la caracterización mucho más detallada (por ejemplo de concentraciones) que permitirá un entendimiento mayor de los flujos densos, uno de los fenómenos naturales más amenazantes en algunas regiones del mundo.

\subsection{Uso de LSPIV para optimizar el funcionamiento de infraestructura hídrica en es- tructuras de descarga de presas}

En el 2011, se utilizó por última vez el vertedero del dique Cabra Corral. Durante este año se registró con un video el momento en el cual se procedió a la apertura de sus compuertas.

En las siguientes figuras se presenta una imagen del video captado y el campo de velocidad superficial promedio estimado con la técnica experimental: 


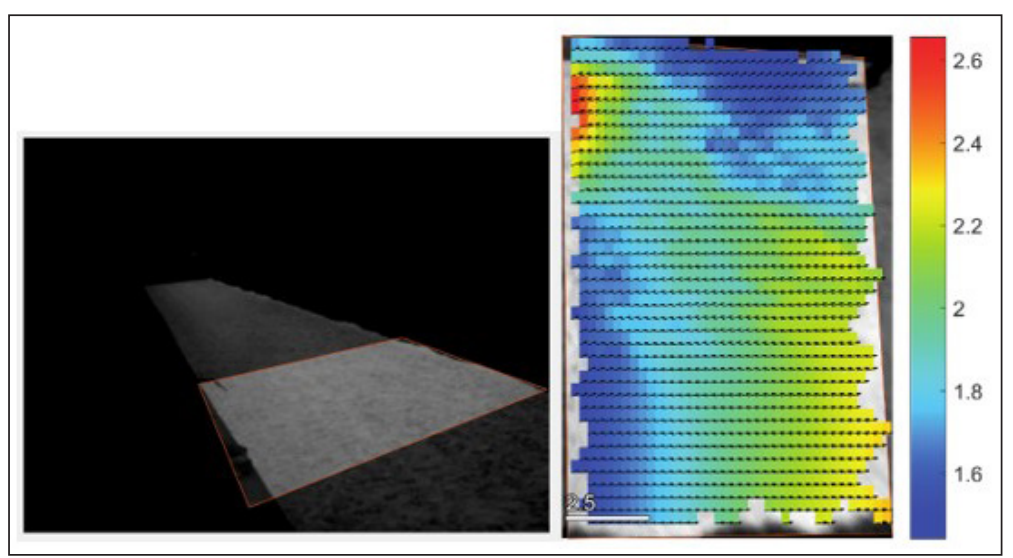

Figura 17: Imagen de la rápida de descarga del vertedero del Cabra Corral y campo de velocidades medias superficiales estimado durante la crecida del año 2011

La velocidad media superficial estimada en la rápida del vertedero fue del orden de los 2 a 2,5 $\mathrm{m} / \mathrm{s}$, y esta medición fue posible gracias a la implementación de la técnica experimental LSPIV, ya que de otra manera sería muy difícil (o imposible) poder medir en esta sección de interés.

Además, a la salida de la central hidroeléctrica del dique El Tunal existe un canal de descarga. Durante una visita a la central en el 2019 se procedió a la estimación de las velocidades superficiales aplicando LSPIV. A continuación, se presentan algunos de los resultados obtenidos:

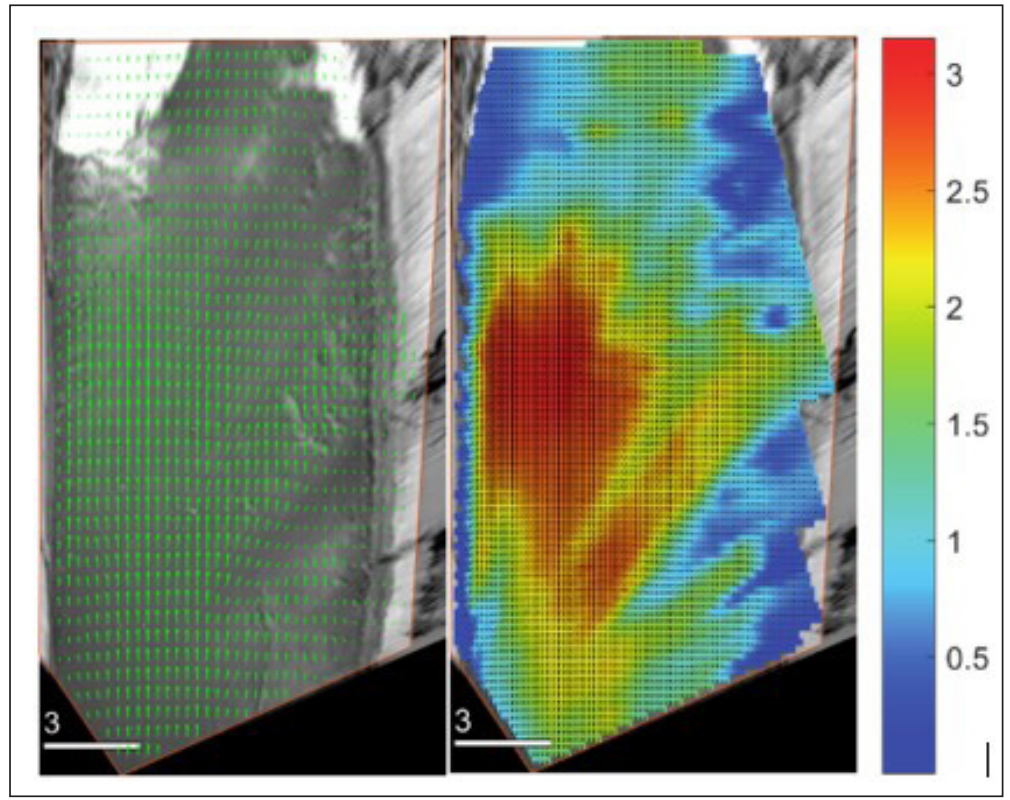

Figura 18: Campo de vectores y de velocidad media superficial en el canal de descarga del dique El Tunal al aplicar LSPIV 
Aplicación de la técnica LSPIV en el diseño hidrológico de infraestructura hídrica

\section{Conclusiones}

Mediante la aplicación de LSPIV, se calibraron modelos hidráulicos y sus respectivos coeficientes de rugosidad durante crecidas en cauces fluviales. Con el registro de videos de crecidas puede estimarse al mismo tiempo las velocidades superficiales del flujo y los niveles alcanzados por el agua, y a partir de esa información calibrarse adecuadamente el modelo. También se ajustaron relaciones $\mathrm{H}-\mathrm{Q}$ en diferentes ríos de la provincia.

Se demostró que LSPIV es una técnica muy adecuada para caracterizar flujos densos y estimar velocidades del frente de estos flujos. Con anterioridad a la realización de este trabajo no era posible medir adecuadamente las velocidades de flujos de este tipo ni siquiera en canales de laboratorio.

Se cuantificó y caracterizó de forma hidrodinámica el flujo escurrido en estructuras de descarga de presas. Es fundamental explicar la importancia de medir las velocidades en flujos de este tipo. Al poder mensurar este parámetro hidráulico se puede verificar el funcionamiento de las estructuras de descarga en prototipo. Esta caracterización experimental en prototipo provee información importante para evaluar el proyecto realizado.

Este trabajo ilustra el gran potencial de las iniciativas de ciencia con participación ciudadana para mejorar la evaluación del riesgo de inundación, ya que los datos hidráulicos, que son tan valiosos, se pueden calcular utilizando mensajes, fotografías y videos producidos por los ciudadanos. Hoy en día, las nuevas tecnologías de comunicación e imágenes digitales han permitido al público producir grandes cantidades de observaciones de inundaciones y compartirlas a través de las redes sociales.

\section{Referencias}

Adrian, R. J. (1984). Scattering particle characteristics and their effect on pulsed laser measurements of fluid flow: speckle velocimetry vs. particle image velocimetry. Appl. Optar. 23, 1690-1691 (1984). Recuperado de https://www.osapublishing.org/ao/abstract. $\mathrm{cfm}$ ?URI $=$ ao-23-11-1690

Adrian, R. J. (1991). Particle-imaging techniques for experimental fluid-mechanics. Annu. Rev. Fluid Mech. 23, 261-304.

Adrian R. J. (2005). Twenty years of particle image velocimetry. Exp. Fluids 39, 159-169.

Bagnold, R. A. (1954). Experiments on a gravity-free dispersion of large solid spheres in a Newtonian Fluid under shear. Proc. R. Soc. London Ser. A. 225:49-63.

Base de Datos Hidrológica Integrada - BDHI. Subsecretaría de Recursos Hídricos. Ministerio del Interior, Obras Públicas y Vivienda. Recuperado de https://www.mininterior.gov.ar/obraspublicas/rh-base.php

Creutin, J. D., Muste, M., Bradley, A., Kim, S. C., and Kruger, A. (2003). River gauging using PIV techniques: a proof of concept experiment on the Iowa River. Fournal of Hydrology, 277(3-4), 182-194.

Fujita, I., Muste, M., and Kruger, A. (1998). Large-scale particle image velocimetry for flow analysis in hydraulic engineering applications. Fournal of Hydraulic Research, 36(3), 397-414. 
Gunawan, B., Sun, X., Sterling, M., Shiono, K., Tsubaki, R., Rameshwaran, Knight, Chandler, Tang, Fujita (2012). The application of LSPIV to a small irregular river for inbank and overbank flows. Flow. Meas. Inst., 24, 1-12.

Johnson, A. M. (1970). Physical Processes in Geology. San Francisco: Freeman.

Le Coz, J., Jodeau Magali, Hauet, A., Marchand, B., and Le Boursicaud, R. (2014). Image-based velocity and discharge measurements in field and laboratory river engineering studies using the free FUDAA-LSPIV software. River Flow, Lausanne, Switzerland.

Ministerio del Interior, Obras Públicas y Vivienda. Base de Datos Hidrológica Integrada-BDHI. Subsecretaría de Recursos Hídricos. Recuperado de https://www.mininterior.gov.ar/obraspublicas/rh-base.php

Muste, M., Fujita, I., and Hauet, A. (2008). Large-scale particle image velocimetry for measurements in riverine environments. Water Resources Research, 44, 1-14.

Patalano, A., García, C. M., \& Rodríguez, A. (2017). Rectification of Image Velocity Results (RIVeR): A simple and user-friendly toolbox for large scale water surface Particle Image Velocimetry (PIV) and Particle Tracking Velocimetry (PTV). Computers E Geosciences, 109, 323-330.

Takahashi, T. (1981). Debris flow. Annual review of fluid mechanics, 13(1), 57-77.

Thielicke, W. and Stamhuis, E. J. (2014). PIVlab - Towards User-friendly, Affordable and Accurate Digital Particle Image Velocimetry in MATLAB. Fournal of Open Research Software 2(1):e30, DOI: http://dx.doi.org/10.5334/jors.bl.

Yano, K., Daido, A. (1965). Fundamental study of mudflow. Bull. Disaster Prevo Res. Inst. Kyoto Univ. 14:69-83. 


\section{Myriam Cecilia Botelli}

Ingeniera Hidráulica, egresada de la Universidad Nacional de Salta. Especialista en Formulación y Evaluación de Proyectos de Inversión. Encargada del Gabinete de Hidráulica e Instalaciones Sanitarias de la Universidad Católica de Salta. Docente de las cátedras de Hidrología y de Hidráulica Aplicada en la carrera de Ingeniería Civil de la Universidad Católica de Salta. Investigadora del I.Es.I.Ing de la Facultad de Ingeniería de UCASAL, Consultora Independiente de Proyectos Hidráulicos, Evaluación de Impacto Ambiental y de Proyectos de Inversión.

cbotelli@ucasal.edu.ar

\section{Nicolás Federico Guillén}

Ingeniero Civil (Universidad Nacional de Córdoba). Doctor en Ciencias de la Ingeniería, Máster en Ciencias de la Ingeniería - Mención Recursos Hídricos (Universidad Nacional de Córdoba). Investigador Visitante del United States Geological Survey, Office of Surface Water (Estados Unidos). Profesor Asistente en la Universidad Nacional de Córdoba. Docente de posgrado en la Especialización en Hidráulica, Maestría en Ciencias de la Ingeniería - Mención Recursos Hídricos y Doctorado en Ciencias de la Ingeniería. Asesor del Secretario de Recursos Hídricos y Coordinador técnico del Plan Integral de Manejo de Embalses en el Ministerio de Servicios Públicos de la Provincia de Córdoba.

nfguillen@hotmail.com

\section{Carlos Marcelo García}

Ingeniero Civil de la Universidad Católica de Córdoba. Máster en Ciencias de la Ingeniería Mención Recursos Hídricos de la Facultad de Ciencias Exactas, Físicas y Naturales de la Universidad Nacional de Córdoba. PhD (Universidad de Illinois en Urbana, Champaign, Estados Unidos). Investigador independiente, Carrera del investigador científico y tecnológico del CONICET. Docente-Investigador de grado y posgrado de la Facultad de Ciencias Exactas, Físicas y Naturales. Universidad Nacional de Córdoba y otras universidades.

cgarcia2mjc@gmail.com

\section{Antoine Patalano}

Perfil académico-profesional.

Doctor en Ciencias de la Ingeniería. Investigador Asistente del CONICET. Desarrollador de RIVeR (https://riverdischarge.blogspot.com/). Docente de Hidrología en la Universidad Nacional de Córdoba.

antoinepatalano@gmail.com 
M. C. Botelli et al.

\section{Marcelo Chalabe}

Ingeniero Civil (Universidad Católica de Salta). Docente de las cátedras de Hidrología y de Hidráulica Aplicada en la carrera de Ingeniería Civil de la Universidad Católica de Salta. Integrante del grupo de investigación Riesgo Hídrico del Instituto de Estudios Interdisciplinarios de Ingeniería (I.Es.I.Ing).

mchalabe@ucasal.edu.ar 